\section{Enfermedades emergentes no infecciosas}

\section{Ezequiel Consiglio ${ }^{1}$}

Forma de citar: Consiglio E. Enfermedades emergentes no infecciosas. Rev Panam Salud Publica. 2008;24(5):361-8.

Palabras clave: enfermedades emergentes, enfermedades no transmisibles, determinantes sociales.

\footnotetext{
1 Departamento de Salud Pública, Facultad de Medicina, Universidad de Buenos Aires. Dirección para la correspondencia: Escuela de Salud Pública, Marcelo T. de Alvear 2202, Buenos Aires C1122AAJ, Argentina. Teléfono/fax (+54 11) 4508-3666. Correo electrónico: econsiglio@intramed.net
}

En años recientes se han definido como enfermedades emergentes aquellas ocasionadas tanto por gérmenes recientemente descritos, como por patógenos previamente conocidos pero que incrementaron marcadamente su frecuencia o modificaron su presentación y evolución clínicas. Estos últimos suelen corresponder a microorganismos que evidenciaron un aumento de su virulencia o de su resistencia a fármacos que hasta ese momento resultaban eficaces para su tratamiento $(1,2)$.

Entre los agentes de reciente descripción se pueden mencionar el virus de la inmunodeficiencia humana (VIH) (3) y la cepa Andes (4) del género Hantavirus, causante del síndrome pulmonar por Hantavirus en la República Argentina y en Chile. Las formas multirresistentes de Mycobacterium tuberculosis (5) y la cepa HO157 de Escherichia coli (6) — causante de una alta proporción de casos de síndrome urémico hemolítico (SUH) — son microorganismos patógenos previamente conocidos que modificaron sus características clínico-epidemiológicas. La enumeración de los agentes infecciosos emergentes sería muy extensa y no es objeto del presente trabajo.

Las enfermedades emergentes de origen infeccioso representan una amenaza a la salud pública, ya sea por la falta de tratamientos específicos (7) o por la eventual sobrecarga que pueden causar en los sistemas de salud. Son también motivo de reciente preocupación por su potencial utilización criminal como armas biológicas (8). En cualquiera de esos potenciales escenarios, el incremento de la morbilidad y de la mortalidad causadas por una enfermedad emergente puede determinar un aumento de la necesidad de recursos e insumos de los sistemas de salud $(9,10)$.

Considerando las características hasta aquí descritas de estas enfermedades, surge la hipótesis de que el universo de estas enfermedades emergentes habría de ampliarse a diversas entidades gnoseológicas no transmisibles.

El objetivo de este trabajo es presentar las características más importantes de las enfermedades emergentes, describir la existencia de enfermedades emergentes de origen no infeccioso y discutir qué ventajas aportaría su identificación como tales. Asimismo, procedería plantear una definición más amplia de enfermedad emergente.

\section{MARCO HISTÓRICO DE LAS ENFERMEDADES EMERGENTES}

En la historia occidental hay al menos dos situaciones en las que se produjo la aparición de lo 
que hoy conocemos como enfermedades emergentes infecciosas. Quizá la más dramática de ellas, por su impacto inmediato y sus consecuencias directas, fue la diseminación de la viruela, enfermedad que diezmó las poblaciones indígenas azteca e inca durante la conquista española de América en los inicios del siglo XVI. La ausencia de inmunidad de estas poblaciones originarias se ha asociado a la gravedad clínica alcanzada toda vez que se expusieran a soldados enfermos de las tropas conquistadoras. Más aún, se ha descrito que cuando los jefes de las tropas conquistadoras repararon en que la enfermedad alcanzaba una letalidad superior entre los indios, utilizaron ropas con secreciones de lesiones variólicas, pertenecientes a sus soldados enfermos, para propiciar el contagio a los indios, en una actitud similar a lo que hoy se entiende por guerra bacteriológica o ataque bioterrorista $(11,12)$.

La otra situación que aparece en un marco histórico de emergencia de enfermedades correspondió a las grandes epidemias registradas durante la Edad Media y la Edad Moderna en las ciudades europeas. Estas epidemias se produjeron en un escenario determinado por el crecimiento de las ciudades sin normas de higiene ni planificación urbana preestablecidas, por migraciones masivas desde el campo originadas a partir de los cambios en los sistemas de producción económica $(13,14)$. Asimismo, esta superpoblación repentina produjo cambios en los comportamientos sociales, merced a los cuales se incrementó abruptamente la exposición a agentes transmisibles en poblaciones hacinadas y sin inmunidad previa. A esta época corresponden las grandes epidemias de peste, cólera y tifus, así como el establecimiento de la endemicidad de la sífilis y de la lepra (15) y el cambio del patrón de transmisibilidad de la tuberculosis, de la vía digestiva a la vía aérea (16).

Estos dos ejemplos históricos dan cuenta del valor que pueden alcanzar distintos fenómenos de cambio social vinculados entre sí y con el proceso de emergencia; y cómo la evolución de los sistemas económico-políticos favoreció este resultado mediante procesos de migración interna, ad intra, y de colonización ad extra al establecer vínculos entre distintas regiones.

\section{DEFINICIÓN DE ENFERMEDAD EMERGENTE Y NECESIDAD DE MAYOR ESPECIFICIDAD}

Los términos "enfermedades infecciosas emergentes", "reemergentes" o simplemente "enfermedades emergentes" suelen utilizarse como sinónimos en la literatura científica. La breve historia del concepto de enfermedad emergente y del uso de los términos citados no ha permitido que se desarrollen matizaciones o diferenciaciones más precisas en el uso de esta terminología (17). Más allá de la utilización de distintos términos, parece haber consenso entre diferentes autores en las características de este tipo de enfermedades (1, 2, 7-10).

La emergencia de enfermedades infecciosas se ha descrito como el resultado de la acción de diversos factores ligados entre sí y que actúan en diferentes niveles de organización, tanto biológica como social $(2,6)$. Al respecto, existen modelos explicativos del proceso salud-enfermedad que usualmente no han sido propuestos para estas enfermedades en particular y que, sin embargo, podrían ser útiles para comprender mejor el mecanismo de emergencia. En el "modelo de cajas chinas" que se aplicó inicialmente a enfermedades no trasmisibles, el resultado de salud o enfermedad depende de la interacción de elementos que se disponen en tres niveles: un nivel macro correspondiente al ámbito medioambiental (social y ecológico); un nivel individual correspondiente a la organización biológica del individuo y a sus aspectos conductuales; y finalmente, un nivel micro, correspondiente a la información genética y el perfil molecular. En los tres niveles del sistema hay interacciones transversales entre los elementos de un mismo nivel e interacciones verticales entre elementos de distintos niveles (18). De similar manera suele describirse la relación entre los factores que determinan la emergencia.

En Estados Unidos, el Instituto de Medicina y los Centros para el Control de Enfermedades definen a las enfermedades emergentes como enfermedades "cuya incidencia se ha incrementado en las dos últimas décadas o amenaza con aumentar en un futuro cercano" 2 (1). Otros autores se refieren a las enfermedades emergentes como enfermedades "nuevamente aparecidas o resurgidas" (8), o como enfermedades "que han aparecido abruptamente o de manera inesperada", o que "aumentan rápidamente su incidencia o el ámbito geográfico en el que se presentan" (2). En síntesis, las características de las enfermedades emergentes enunciadas son: a) su origen infeccioso; b) un aumento de su incidencia, que a su vez, puede ser abrupto; y c) una categorización como amenaza o como fenómeno inesperado. Sin embargo, el incremento de la incidencia de una enfermedad define una epidemia, o una pandemia si la enfermedad se extiende a todo el planeta, pero no representa una característica específica del carácter emergente, sino un aspecto descriptivo del mismo. Por otro lado, la relación entre el carácter emergente y una zona geográfica o una

\footnotetext{
2 Cuando se citan en castellano artículos en inglés, la traducción es del autor de este trabajo.
} 
población hasta ese momento no afectada también puede ser comprendida como categoría descriptiva. Por último, la asociación entre carácter emergente y la amenaza "de que se incremente su frecuencia de aparición en los próximos años" $(1,10)$ cae en una subjetividad que parece tautológica. Las distintas definiciones mencionadas parecen tener así un carácter marcadamente descriptivo.

Si bien hay autores que adjudican un valor explicativo a la propia descripción de los fenómenos (19), eso no parece ser suficiente en el caso de las enfermedades emergentes.

Otras disciplinas pueden ayudar en el intento de definición. Desde una perspectiva más amplia y reciente, la física ha coincidido en la preocupación por los fenómenos emergentes. En el marco de un modelo explicativo de sistemas no lineales, esto es, sistemas cuyos resultados son difícilmente predecibles, se define el concepto de emergencia como la aparición de fenómenos cualitativamente inesperados que resultan de interacciones locales y suelen producirse de modo abrupto (20). Aún cuando esta definición viene de una disciplina que critica los modelos deterministas, más próximos a los utilizados en las ciencias de la salud, parece tener un carácter más específico puesto que considera dos hechos que exceden el marco de lo descriptivo: que se trata de resultados usualmente abruptos pero característicamente inesperados, y que surgen como resultado de interacciones locales. El origen local de dichas interacciones tampoco parece ser menor puesto que en la literatura se suelen puntualizar estos escenarios locales en la presentación de enfermedades emergentes.

\section{ENFERMEDADES EMERGENTES DE ORIGEN NO INFECCIOSO}

El objetivo de este trabajo no es hacer una lista de posibles enfermedades emergentes no infecciosas, sino plantear su existencia. Para ello se presentarán algunos casos ilustrativos y se discutirán algunas enfermedades cuya frecuencia o importancia se ha generalizado, sin que por ello dejen de expresar interacciones locales en el origen de su emergencia.

La dimensión psicológica de la salud, postulada en la propia definición de salud de la OMS (21), ha logrado un reconocimiento amplio en las agendas de salud pública durante las últimas décadas. Las estimaciones de años de vida ajustados según discapacidad (AVAD, o QALY según las iniciales de la expresión inglesa) que el estudio de Carga Mundial de Enfermedad (22) atribuye a los trastornos mentales dan cuenta de esa importancia. Así, en los países de rentas bajas y medias, la sép- tima causa de AVAD perdidos corresponde a la depresión mayor y trastornos conexos, mientras que en los países de rentas altas, esta misma causa ocupa el tercer puesto en importancia. La enfermedad de Alzheimer y otras demencias, que pueden caracterizarse en conjunto como trastornos cognitivos, ocupan el cuarto lugar y las consecuencias del abuso del alcohol el noveno (23). Se prevé que la depresión mayor será la principal causa de discapacidad para el año 2020 en todo el mundo, fundamentalmente por el aumento de su importancia en los países denominados en desarrollo (24). En este sentido, de acuerdo con estimaciones basadas en trabajos de campo, en América Latina y el Caribe más de 12 millones de personas padecen depresión mayor, al menos 8 millones presentan alguna consecuencia del abuso del alcohol y algo más de 6 millones padecen trastornos bipolares, ansiedad generalizada o trastornos de pánico (25).

Los trastornos mentales mencionados representan un motivo de preocupación por sus consecuencias individuales y colectivas. A ellos deben añadirse los suicidios, situados en un espacio de intersección taxonómica con las violencias, y también algunos trastornos nutricionales ligados a la conducta, como la anorexia nerviosa y la bulimia.

Se ha estimado que el suicidio fue la causa de muerte de más de 800000 personas en el año 2000, lo que supone una tasa anual mundial de 145 suicidios por millón de personas (26). En países de rentas altas se han observado incrementos de hasta $50 \%$ en la tasa de suicidio en un período reciente de 10 años (27) y se prevé que los suicidios sigan en aumento en todo el mundo (24). En el incremento de la frecuencia del suicidio podrían influir diversos factores tales como las situaciones de violencia de diverso origen (familiar, laboral), los factores socioeconómicos —entre los que destaca la pobreza- y las comorbilidades con trastornos crónicos como la infección por VIH/sida y la depresión (28).

Existe un hecho distintivo en la valoración del suicidio como fenómeno emergente: su frecuencia cada vez mayor a edades tempranas, tanto en países de rentas altas (29) como en naciones de rentas medias o bajas (30). El aumento de frecuencia de los suicidios de adolescentes y jóvenes representa un hecho reciente e inesperado que confiere al suicidio una complejidad y un dramatismo hasta ahora no reconocidos.

También entre los más jóvenes se dan los dos trastornos psíquico-nutricionales ya mencionados, la anorexia nerviosa y la bulimia (31).

Según algunos autores la frecuencia de la anorexia nerviosa parece ser más o menos estable (32, 33), pero otros investigadores han observado una incidencia con una clara tendencia al aumento durante las últimas décadas (34). Este trastorno afecta 
con una cierta selectividad a mujeres adolescentes y jóvenes pertenecientes a grupos sociales no vulnerables económicamente $(33,35)$. Esta característica es distintiva de este tipo de entidades. También parece haber un aumento en la incidencia de la bulimia (36), otro trastorno de la alimentación ligado al psiquismo en cuya patogenia se han postulado factores sociales, como los modelos de belleza occidentales, que actuarían como estímulo para la expresión generalizada de características individuales que llevarían al desequilibrio nutricional (37).

En otro extremo, la obesidad también representaría una situación emergente como resultado de la interacción de factores físicos, psíquicos y sociales. El término ya habitual "epidemia de obesidad" da cuenta de la percepción del incremento de la obesidad $(38,39)$, que ya ha despertado interés por su presentación a edades en las que era muy infrecuente hace pocas décadas (40).

Desde el punto de vista causal, el sobrepeso y la obesidad corresponden al resultado de una verdadera transición nutricional caracterizada por un incremento en la ingesta de grasas y alimentos azucarados (41), en un contexto de desarrollo tecnológico en todos los órdenes de la vida que, a su vez, favorece el sedentarismo. Este sedentarismo, además, puede relacionarse con el desarrollo en los últimos tiempos de un modelo de urbanización que se ha visto acompañado de pérdida del espacio público donde transitar o recrearse, de nuevas conductas condicionadas por los requerimientos laborales que resultan en la disminución de la actividad física y, además, de la menor accesibilidad de los alimentos de buena calidad — que tienen menos contenido calórico - en poblaciones cada vez más condicionadas económicamente (42). El aumento de la prevalencia de sobrepeso y obesidad se ha observado en diversos países de rentas altas. En Estados Unidos, por ejemplo, la obesidad, objetivada mediante un índice de masa corporal de 30 ó más, afectó en 1999-2002 a 30.4\% de la población adulta de 20 o más años de edad (43). Pero el aumento de la prevalencia de sobrepeso y obesidad se ha observado también en países de rentas medias o bajas $(44,45) \mathrm{y}$, por otro lado, se han descrito cambios en los grupos etarios afectados y en los perfiles de género y renta: ha aumentado la frecuencia de estos trastornos en niños y adolescentes y el exceso de peso es más frecuente entre los más pobres, y en las niñas más que en los niños $(46,47)$. Además, en el marco de los riesgos asociados al proceso de transición epidemiológica, la expansión del sobrepeso y la obesidad actúa, como el tabaquismo, como factor sinérgico de riesgos para enfermedades cardiovasculares ya establecidos, generándose así grupos de personas particularmente vulnerables (48). También se ha descrito la contribución del exceso de peso al agravamiento de las lesiones óseas y de los tejidos blandos, traumáticas o no traumáticas, en diferentes localizaciones del aparato locomotor (49). Esta sinergia de factores de riesgo y condiciones clínicas preexistentes dificulta aún más el control de dichas enfermedades.

Las lesiones externas, también incluidas bajo la genérica denominación de violencias, incluyen causas y mecanismos de enfermedad y muerte de diversa índole, intensidad y repercusión. Así, en un marco de notoria heterogeneidad, dentro de esta categoría se incluyen los homicidios, los suicidios, las defunciones o las lesiones no mortales por choques o atropellos automovilísticos y otras lesiones que pueden ser producidas por diversos agentes, por ejemplo en el domicilio o en el ámbito laboral. Las muertes y las lesiones no mortales por arma de fuego $\mathrm{u}$ otros tipos de causas asociadas a conflictos bélicos no suelen ser consideradas en términos de salud pública, pero recientes análisis han dado cuenta de su importancia directa e indirecta en este campo (50).

Las defunciones y los traumatismos no mortales relacionados con el tránsito automovilístico representan un problema de salud pública cuya trascendencia fue puesta de relieve por la decisión de la OMS de considerarlos como su tema principal durante el año 2004 (51). Las estimaciones de carga mundial de enfermedad provocada por traumatismos relacionados con el tránsito llegan a 1,2 millones de muertes anuales y 50 millones de lesiones de gravedad variable; los costos globales estimados para esta carga de mortalidad y morbilidad superan los 5000 millones de dólares estadounidenses (52). En términos de evolución histórica se ha registrado, por un lado, un descenso de la mortalidad por este tipo de accidentes en países desarrollados, y por otro, un incremento en los países de Asia, América Latina y el Caribe y África, aún cuando existen datos menos fiables en estos últimos pese a ser los más afectados. Las peores tasas de mortalidad corresponderían a países latinoamericanos: 41,7 por 100000 habitantes en El Salvador y 41,0 en la República Dominicana en el año 2002 (53).

Las enfermedades profesionales fueron descritas hace ya cuatro siglos por Bernardino Ramazzini (54), pero en la última década estas enfermedades y las lesiones y traumatismos en el medio laboral han comenzado a ser vistos con mayor interés y preocupación debido a su aumento y a sus consecuencias de orden social.

Se ha estimado mediante métodos directos e indirectos que las exposiciones laborales son responsables de unos 100 millones de lesiones anuales en todo el planeta, de las que unas 100000 concluyen en la muerte del trabajador. Además, se producirían más de 10 millones de casos nuevos de enfer- 
medades profesionales, de los que unos 700000 casos concluirían en la muerte de la persona afectada (55). También se ha estimado que los riesgos profesionales en su conjunto fueron responsables de $37 \%$ de las dorsalgias, $16 \%$ de las pérdidas auditivas, $13 \%$ de los diagnósticos de enfermedad pulmonar obstructiva crónica, $11 \%$ de los casos de asma, 9\% de los cánceres de pulmón y $2 \%$ de las leucemias, así como la práctica totalidad de los casos de neumoconiosis y mesotelioma (56).

Se han descrito diversos factores de riesgo que explicarían el incremento de la carga de enfermedad ligada a las exposiciones laborales. La heterogeneidad de estos factores de riesgo puede ilustrarse por ejemplo por los factores psicosociales a los que se atribuye en años recientes una creciente demanda de asistencia por violencia y abuso en el ambiente laboral en algunos países desarrollados (57); o por los agentes químicos como el asbesto, cuya exposición laboral provoca mesotelioma pleural y ha sido objeto de medidas de vigilancia específicas en años recientes (58). Estas enfermedades profesionales y laborales también pueden considerarse enfermedades emergentes.

Finalmente, es necesario considerar que, aparte de las acciones indirectas de los factores ambientales, que pueden influir de forma más o menos indirecta en las enfermedades anteriormente mencionadas, se ha descrito el incremento de enfermedades directamente ligadas a modificaciones específicas del medio ambiente natural. Tal es el caso del melanoma, cuya incidencia aumenta con el incremento de la exposición a radiación ultravioleta natural que, a su vez, está relacionada con la disminución de la capa estratosférica de ozono $(59,60)$. Otro ejemplo es el asma bronquial, cuya emergencia se describió inicialmente en países desarrollados, en los que se controló luego mediante tratamientos farmacológicos, para emerger recientemente en países de rentas medias y bajas (61). El impacto directo de los cambios ambientales representa, en una escala más amplia, el resultado del comercio, el transporte y la actividad industrial en el marco mundial de globalización económica. Pasos intermedios en las vías causales de estos procesos pueden ser, además de la mencionada pérdida de la capa de ozono, el calentamiento atmosférico, la creciente presencia de contaminantes químicos, la pérdida de biodiversidad, la proliferación de especies invasoras y el deterioro de los ecosistemas en general (62).

\section{DISCUSIÓN}

Los casos presentados muestran que las características clásicas atribuidas a las enfermedades infecciosas emergentes - aumento de frecuencia o extensión a nuevas poblaciones en épocas recientes y representando una amenaza para la salud o la seguridad pública $(2,7-10)$ - también se dan en enfermedades no trasmisibles. Pero muchas de estas son también resultado de complejas relaciones multifactoriales que entran en juego mediante interacciones locales, determinando un resultado inesperado. Que un resultado sea cualitativamente inesperado implica que no es previsible hasta que aparece. De los ejemplos considerados, la obesidad o los traumatismos relacionados con el tránsito automovilístico han sido señalados por algunos autores como males endémicos, sin embargo resultan inesperados en su frecuencia, en su impacto, o en las poblaciones que están afectando.

A partir de estas características comunes queda trazada una analogía entre, por ejemplo, la emergencia y expansión de las infecciones por distintas especies del género Enterococcus $(63,64)$ y la aparición de adolescentes obesos en comunidades de rentas bajas; o entre la infección por $\mathrm{VIH} /$ sida y ciertas lesiones musculoesqueléticas, como el síndrome del túnel carpiano, relacionadas con la generalización de tecnologías antes inexistentes, como las computadoras personales (65). Más aún, el postulado general de que la emergencia de las enfermedades se produce como resultado de una compleja interacción entre factores ambientales, sociales y poblacionales (66) difícilmente puede ceñirse exclusivamente a las enfermedades ocasionadas por agentes infecciosos, cuando la mayor carga de enfermedad ligada a las enfermedades y trastornos que se han discutido, corresponde a enfermedades no trasmisibles (22).

La emergencia de enfermedades no infecciosas debe interpretarse, a su vez, en un escenario de transición epidemiológica. Paul Farmer (17) asegura que dicho modelo permite prever el modo de enfermar y morir que tienen los habitantes de los países desarrollados en los que este proceso se ha llevado a cabo plenamente. En este sentido, así como en ese modelo de transición no se previó el recrudecimiento de algunas enfermedades infecciosas, ni la aparición de nuevos gérmenes patógenos, tampoco se previó la aparición o expansión de enfermedades como las discutidas en este trabajo. Puede entonces interpretarse que dicha emergencia expresa la acción de diversos factores cuya calidad, intensidad e interrelación fueron suficientes para causar una nueva enfermedad. Y, a su vez, estas enfermedades emergentes pueden considerarse un grupo de eventos adversos consecuencia de los procesos de industrialización, post-industrialización y mundialización ligados a los avances científico-tecnológicos. Procesos que, junto con el saneamiento ambiental, la mejora de la nutrición y la expansión del uso de vacunas contra las enfermedades epidé- 
micas de la infancia determinaron el proceso de transición epidemiológica mencionado. Estos efectos, si bien no anulan los resultados positivos hasta ahora obtenidos, advierten sobre un nuevo perfil de exposiciones, riesgos y resultados en materia de salud y enfermedad. En ese sentido, la existencia de las enfermedades emergentes no infecciosas apunta a los efectos generales que han tenido sobre la naturaleza los sistemas de producción económica en donde priman la eficiencia y la acumulación en detrimento de un desarrollo sustentable con un marco de equidad.

En este trabajo se han presentado datos y características mundiales o regionales de las enfermedades que se han discutido a modo de ejemplo, aún cuando se hayan postulado los ámbitos locales para valorar su carácter emergente. Esta aparente contradicción queda resuelta en la medida en que estos casos no se han utilizado para hacer una enumeración de enfermedades emergentes, sino para cuestionar que estas sean de origen exclusivamente infeccioso. Ante estos casos, considerados globalmente, la pregunta que debe plantearse es: ¿cuál es la diferencia entre un proceso de emergencia y otro de simple diseminación de una enfermedad en forma de brote o epidemia? Aunque la diferenciación de ambos fenómenos no puede ser estricta, habría que subrayar que una enfermedad emergente representa un hecho cualitativamente inesperado. Pero además, se puede observar un gradiente de complejidad que hace que este proceso sea diferente a los mencionados en los que predomina solamente el aumento de frecuencia. Este gradiente de complejidad implica la interrelación de los factores que intervienen en cada uno de estos resultados, y también se explica por los diferentes requerimientos que tienen un brote o una epidemia frente a una enfermedad emergente, para su control.

La identificación del carácter emergente de una enfermedad debe promover acciones de control no solo en el marco de los servicios de salud sino también en las instituciones encargadas del manejo de los determinantes ambientales, económicos, políticos y sociales que intervinieron en el proceso de emergencia. Podría pensarse que se trata de acciones más amplias que las dispuestas para el control de una epidemia o un brote, cuya aparición dependería, en principio, de un número menor de factores y de una relación de menor complejidad.

En el ámbito específico de los sistemas y servicios de salud, el fin último de la categorización de una enfermedad como emergente consiste en programar acciones de promoción y prevención como estrategia central para el control de las exposiciones e interacciones que llevaron a su emergencia. Esto resultaría de mayor pertinencia en los casos en los que no hay tratamiento definitivo, o este resulta sólo paliativo, como por ejemplo la infección por $\mathrm{VIH} /$ sida entre las enfermedades trasmisibles, o de mesotelioma entre las no trasmisibles, por citar dos ejemplos. Por otro lado, es responsabilidad del sistema de salud poner énfasis en la formación curricular sobre esta temática en las escuelas y facultades de todas las ciencias de la salud. Énfasis que debe traducirse en oportunidad y completitud de los aprendizajes efectuados y de las enseñanzas impartidas. La diseminación de la información referida a los procesos de emergencia de enfermedades también debería incluir a la comunidad, tal como se han planteado algunos autores que proponen un modelo de discusión ampliada de pares para temas de alta complejidad en los que la participación popular resulta decisiva en la identificación y control de noxas (67).

Por último, hay que señalar que las enfermedades emergentes adquieren un valor paradigmático como resultado de modelos complejos. Así, estos desenlaces confieren a la planificación intersectorial una importancia crucial en el control y erradicación de factores sociales estructurales, factores individuales conductuales y factores económicos y políticos que contribuyeron a la aparición de una nueva enfermedad, ya sea por acción directa, indirecta u omisión. Si se circunscribieran exclusivamente al sector salud las tareas de control de factores de exposición ambientales, laborales, ecológicos y urbanos, por ejemplo, los resultados serían ciertamente insuficientes. No sólo por falta de competencia técnica, sino por falta de jurisdicción y por sobrecarga de objetivos sustantivos. La planificación intersectorial parece alcanzar un valor instrumental y operativo difícil de soslayar en la organización comunitaria.

A partir de la identificación de enfermedades emergentes no transmisibles, y una vez explicitada la importancia de su identificación como tal, se debería avanzar en una definición más amplia y pertinente. De acuerdo con lo presentado y discutido en este trabajo, una definición de las enfermedades emergentes que las describa y explique debería incluir al menos que se trata de procesos, transmisibles o no, que se dieron de novo o incrementaron su frecuencia durante los últimos años, y que afectaron nuevas poblaciones o modificaron su presentación clínica de modo inesperado como resultado de interacciones multifactoriales complejas de desarrollo local.

Agradecimiento. El autor desea agradecer a Fúlvio Nedel sus observaciones y comentarios sobre este trabajo. 


\section{SYNOPSIS}

\section{Emerging noninfectious diseases}

In recent years, emerging diseases were defined as being infectious, acquiring high incidence, often suddenly, or being a threat or an unexpected phenomenon. This study discusses the hallmarks of emerging diseases, describing the existence of noninfectious emerging diseases, and elaborating on the advantages of defining noninfectious diseases as emerging ones. From the discussion of various mental health disor- ders, nutritional deficiencies, external injuries and violence outcomes, work injuries and occupational health, and diseases due to environmental factors, the conclusion is drawn that a wide variety of noninfectious diseases can be defined as emergent. Noninfectious emerging diseases need to be identified in order to improve their control and management. A new definition of "emergent disease" is proposed, one that emphasizes the pathways of emergence and conceptual traits, rather than descriptive features.

Key words: emerging diseases, noninfectious diseases, social determinants.

\section{REFERENCIAS}

1. Centers for Diseases Control and Prevention $(\mathrm{CDC})$. Addressing emerging infectious diseases threat: a prevention strategy for the United States. Atlanta, GA: US Department of Health and Human Services, Public Health Service; 1994.

2. Morse SS. Factors in emergence of infectious diseases. Emerg Infect Dis. 1995;1: 7-15.

3. Gottlieb MS, Schroff R, Schanker HM, Weisman JD, Fan PT, Wolf RA et al. Pneumocystis carinii pneumonia and mucosal candidiasis in previously healthy homosexual men. Evidence of a new acquired cellular immunodeficiency. N Engl J Med. 1981;305:1425-31.

4. Lázaro ME, Resa AJ, Barclay CM, Calanni L, Samengo L, Martinez L et al. Síndrome pulmonar por hantavirus en el Sur andino argentino. Medicina (Buenos Aires). 2000;60:289-301.

5. Villarino ME, Geiter LJ, Simone PM. The multidrug-resistant tuberculosis challenge to public health efforts to control tuberculosis. Public Health Rep. 1992; 107:616-25.

6. Tenover FC, Hughes JM. Challenges of emerging infectious diseases. JAMA. 1996;275:300-4.

7. Lederberg J. Infectious diseases as an evolutionary paradigm. Emerg Infect Dis. 1997;4:417-27.

8. Centers for Diseases Control and Prevention. Preventing emerging infectious diseases. A strategy for the 21st century. Atlanta, GA: US Department of Health and Human Services; 1998.

9. Satcher D. Emerging infections: getting ahead of the curve. Emerg Infect Dis. 1995;1:1-6.

10. Epstein PR. Emerging diseases and ecosystem instability: new threats to public health. Am J Pub Health. 1995;85: 168-72.

11. Diomedi PA. La guerra biológica en la conquista del nuevo mundo: una revisión histórica y sistemática de la literatura. Rev Chil Infect. 2003;20:19-25. sity Press; 1991.
12. García Cáceres U. La implantación de la viruela en los Andes, la historia de un Holocausto. Rev Peru Med Exp Salud Publica. 2003;20:41-50.

13. Livi Bacci M. Population and nutrition: An essay on European demographic history (trad. de T. Croft-Murray y C. Pisen). New York: Cambridge Univer-

14. Engels F. La situación de la clase obrera en Inglaterra. Diáspora. Buenos Aires, 1974.

15. Watts S. Epidemias y poder: Historia, enfermedad, imperialismo (trad. de C. Gardini). Andrés Bello, Barcelona, 2000.

16. Bates JH \& Stead W W. The history of tuberculosis as a global epidemic. Medical Clinics of North America. 1993; 77(6):1205-17.

17. Farmer P. Social inequalities and emerging infectious diseases. Emerg Infect Dis. 1996;2:259-69.

18. Susser M, Susser E. Choosing a future for epidemiology: II. From black box to Chinese boxes and eco-epidemiology. Am J Public Health. 1996;86:674-7.

19. El proceso de la investigación y sus dimensiones. En: Samaja J. Epistemología y metodología; $3^{\mathrm{a}}$ ed. Eudeba. Buenos Aires, 2006.

20. Goldberger AL. Complex Systems. Proc Am Thorac Soc. 2006;3:467-72.

21. OMS -Organización Mundial de la Salud-Preámbulo a la Constitución de la Organización Mundial de la Salud, adoptada por la Conferencia Sanitaria Internacional Salud. Nueva York, 19 de junio-22 de julio, 1946.

22. Murray CJL, Lopez AD. Alternative projections of mortality and disability by cause 1990-2020: Global Burden of Disease Study. Lancet. 1997;349:1498-504.

23. Lopez AD, Mathers CD, Ezzati M, Jamison DT, Muray CJL. Global and regional burden of disease and risk factors, 2001: systematic analysis of population health data. Lancet. 2006;367:1747-57.

24. Murray CJL, Lopez AD. Mortality by cause for eight regions of the world:
Global Burden of Disease Study. Lancet. 1997;349:1269-76.

25. Kohn R, Levav I, Caldas de Almeida JM, Vicente B, Andrade L, et al. Los trastornos mentales en América Latina y el Caribe: asunto prioritario para la salud pública. Rev Panam Salud Publica. 2005;18: 229-40.

26. World Health Organization. Injury: a leading cause of the global burden of disease. Ginebra, 1999 (WHO/HSC/ PVI/99.11).

27. Ruiz-Pérez I, Olry de Labry-Lima. El suicidio en la España de hoy. Gac Sanit. 2006;20(supl.1):25-31.

28. Krug EG, Dahlberg LL, Mercy JA, Zwi $A B$, Lozano $R$, eds. La violencia autoinfligida. En: Organización Panamericana de la Salud. Informe mundial sobre la violencia y la salud. Washington, DC OPS, 2003 (Publicación Científica y Técnica No. 588, pp. 201-31)

29. Gunnell D, Middleton N, Whitley e, Dorling D, Frankel S. Why are suicide rates rising in young men but falling in the elderly?-A time-series analysis of trends in England and Wales 1950-1998. Social Sci Med. 2003;57:595-611.

30. Cendales R, Vanegas C, Fierro M, Córdoba R, Olarte A. Suicide trends in Colombia, 1985-2002. Rev Panam Salud Publica. 2007;22:231-8.

31. Fairburn CG, Harrison P J. Eating disorders. Lancet. 2003;361:407-16.

32. van Son GE, van Hoeken D, Bartelds AIM, van Furth EF, Hoek HW. Time trends in the incidence of eating disorders: A primary care study in The Netherlands. Int J Eat Disord. 2006;39: 565-9.

33. Råstam M, Gillberg C, Garton M. Anorexia nervosa in a Swedish urban region. A population-based study. Br J Psychiatry. 1989;155:642-6.

34. Luca AR, Beard CM, O'Fallon WM, Kurland LT. 50-year trends in the incidence of anorexia nervosa in Rochester, Minn: A population-based study. Am J Psychiatry. 1991;148:17-22. 
35. Pawluck DE, Gorey KM. Secular trends in the incidence of anorexia nervosa: integrative review of population-based studies. Int J Eat Disord. 1998;23:347-52

36. Currin L, Schmidt U, Treasure J, Jick H. Time trends in eating disorder incidence. Br J Psychiatry. 2005;18:132-5.

37. Iancu I, Spivak B, Ratzoni G, Apter A, Weizman A. The sociocultural theory in the development of anorexia nervosa. Psychopathology. 1994;27:29-36.

38. Rippe JM. The obesity epidemic: challenges and opportunities. J Am Diet Assoc. 1998;98(suppl 2):S5.

39. Rubenstein AH. Obesity: a modern epidemic. Trans Am Clin Climatol Assoc. 2005;116:103-11.

40. Lowrey GH. Obesity in the adolescent. Am J Public Health Nations Health. 1958;48:1354-8.

41. Hawkes C. Uneven dietary development: linking the policies and processes of globalization with the nutrition transition, obesity and diet-related chronic diseases. Global Health. 2006;28:4

42. Lopez RP, Hynes HP. Obesity, physical activity, and the urban environment: public health research needs. Environmental Health. 2005;5:25

43. Hedley AA, Ogden CL, Jonson CL, Carroll MD, Curtin LR, Flegal KM. Prevalence of overweigth and obesity among US children, adolescents and adults, 1999-2002. JAMA. 2004;291:2847-50.

44. Prentice AM. The emerging epidemic of obesity in developing countries. Int J Epidemiol. 2006;35:93-9.

45. Armstrong MEG, Lambert MI, Sharwood KA, Lambert EV. Obesity and overweight in South African primary school children-The Health of the Nation Study. S Afr Med J. 2006;96: 439-44.

46. Wardle J, Henning Brodersen N, Cole TJ, Jarvis MJ, Boniface DR. Development of adiposity in adolescents: five year longitudinal study of an ethnically and socioeconomically diverse sample of young people in Britain. BMJ. 2006;332: 1130-5.

47. Salazar-Martinez E, Allen B, FernandezOrtega C, Torres-Mejia G, Galal O, Lazcano-Ponce E. Overweight and obesity status among adolescents from Mexico and Egypt. Arch Med Res. 2006; 37:535-42.

48. Healton ChG, Vallone D, McCauslnad KL, Xiao H, Green MP. Smoking, obesity, and their co-ocurrence in the United States: cross sectional analysis. BMJ. 2006;333:25-6.

49. Wearing SC, Henning EM, Byrne NM, Steele JR, Hills AP. Musculoskeletal disorders associated with obesity: a biomechanical perspective. Obes Rev. 2006;3:239-50.

50. Roberts L, Lafta R, Garfield R, Khudhairi J, Burnham G. Mortality before and after the 2003 invasion of Iraq: cluster sample survey. Lancet. 2004;364:1857-64.

51. Día Mundial de la Salud-2004: Seguridad vial. http://www.who.int/worldhealth-day/previous/2004/es/.

52. Peden M, Scurfield R, Sleet $R$, et al. World report on road traffic injury prevention. World Health Organization. Geneva, 2004.

53. Ameratunga $S$, Hijar $M$, Norton $R$ Road-traffic injuries: confronting disparities to address a global-health problem. Lancet. 2006;367:1533-40.

54. Rosen G. A history of public health. Johns Hopkins University Press. Baltimore, 1993.

55. Leigh J, Macaskill P, Kuosma E, Mandryk J. Global burden of disease and injury due to occupational factors. Epidemiology. 1999;10:626-31.

56. Fingerhut $M$, Nelson DI, Driscoll $T$, Concha-Barrientos M, Steenland K, Punnett $\mathrm{L}$ et al. The contribution of occupational risks to the global burden of disease: summary and next steps. Med Lav. 2006;97:313-21.

57. Gilioli R, Campanini P, Fichera GP, Punzi S, Cassitto MG. Emerging aspects of psychosocial risks: violence and harassment at work. Med Lav. 2006;97(2):160-4.
58. NIOSH. The Work-Related Lung Disease Surveillance Report, 2002. NIOSH Publication No. 2003-111, pp. 1-24.

59. Bentham $G$, Aase A. Incidence of malignant melanoma of the skin in Norway, 1955-1989: associations with solar ultraviolet radiation, income and holidays abroad. Int J Epidemiol. 1996;25:1132-8.

60. Jemal A, Devesa SS, Fears TR, Hartge P. Cancer Surveillance Series: Changing Patterns of Cutaneous Malignant Melanoma Mortality Rates Among Whites in the United Status. J Natl Cancer Inst. 2000;92:811-8.

61. Ait-Khaled N, Enarson D, Bousquet J. Chronic respiratory diseases in developing countries: the burden and strategies for prevention and management. Bull World Health Organ. 2001;79:971-79.

62. McMichael AJ, Beaglehole R. The changing global context of public health. Lancet. 2000;356:495-9.

63. Nicas TI, Wu CY, Hobbs JN Jr, Preston DA, Allen NE. Characterization of vancomycin resistance in Enterococcus faecium and Enterococcus faecalis. Antimicrob Agents Chemother. 1989;33:1121-4.

64. Jones ME, Draghi DC, Thornsberry C, Karlowsky JA, Sahm DF, Wenzel RP. Emerging resistance among bacterial pathogens in the intensive care unit: A European and North American surveillance study (2000-2002). Ann Clin Microbiol Antimicrob. 2004;3:14.

65. Juul-Kristensen B, Jensen C. Self-reported workplace related ergonomic conditions as prognostic factors for musculoskeletal symptoms: the "BIT" follow up study on office workers. Occup Environ Med. 2005;62:188-94.

66. Possas CA. Social ecosystem health: confronting the complexity and emergence of infectious diseases. Cad Saude Publica. 2001;17:31-41

67. Funtowicz S, Ravetz JR. Epistemología política: ciencia con la gente. Centro Editor de América Latina. Buenos Aires, 1993. 